American Journal of Pharmacology and Toxicology 1 (3): 48-53, 2006

ISSN 1557-4962

(C) 2006 Science Publications

\title{
Inflammatory Cytokine Expression Patterns in Rat Skin Exposed to JP-8 Jet Fuel
}

\author{
Randle M. Gallucci and Bethany M. Mickle \\ Department of Pharmaceutical Sciences, Health Sciences Center, College of Pharmacy \\ The University of Oklahoma, Oklahoma City, OK, USA
}

\begin{abstract}
One of the main complaints of personnel exposed to JP-8 jet fuel is irritant dermatitis. The purpose of this investigation is to describe the JP-8 induced inflammatory cytokine response in skin over various periods of exposure. JP-8 jet fuel or acetone control $(300 \mu \mathrm{L})$ was applied to the denuded skin of rats once a day for up to seven days. Skin samples from the exposed area were collected after one, three, five and seven days of exposure. RT-PCR was performed utilizing total skin RNA to examine the expression of various inflammatory cytokines. The mRNA's for the chemokines CXCL1, 2, 3 and 10, as well as CCL3, 5, 11 and 20 were variably modulated during the course of exposure, favoring the expression of those that attract PMN. IL- $1 \beta$ and IL-6 mRNA's were significantly induced by five days, while IL-10 was not significantly different from control at any time point. Paradoxically, skin IL-6 protein content was found to decrease continually throughout the time course as determined by ELISA. Data from the present study indicates that repeated JP-8 exposure induces numerous proinflammatory cytokines in skin that tend to favor neutrophil accumulation. The increased expression of these cytokines and chemokines may lead to increased inflammatory infiltrate and injury of the skin, resulting in JP-8 induced irritant dermatitis.
\end{abstract}

Key words: JP-8 jet fuel, dermatitis, cytokines, chemokines, inflammation

\section{INTRODUCTION}

JP-8 is a relatively new jet fuel that the U.S. Air force adopted for use in 1996, replacing JP-4 fuel. As documented on the DoD website, roughly sixty billion gallons of JP-8 are produced annually for various uses, including jets, heaters, stoves, tanks, etc. In addition, JP-8 is very similar to Jet A, which is a commercial jet fuel in common use. While considered safer than its predecessor, JP-8 has been found to adversely affect various tissues including pulmonary ${ }^{[1]}$, immune $e^{[2-4]}$ and $\operatorname{skin}^{[5]}$. While the low volatility of JP-8 was one of the primary reasons for its adoption, this characteristic appears to enhance its toxic effects, perhaps by increasing the duration of dermal exposure ${ }^{[6]}$. Variable transport of JP-8 components occurs across a skin barrier, with aromatic components most rapidly transported $^{[7]}$. Thirteen different components of JP-8 are known to penetrate rat skin, yet only aliphatic components persist long enough to be detected ${ }^{[7]}$. Performance additives contained in JP-8 enhance absorption and modulate retention of aliphatics in $\operatorname{skin}^{[8]}$, thus possibly increasing the irritating effects of these chemicals.

A prominent route of human exposure to JP-8 is via transdermal absorption. One of the purported toxic effects associated with dermal exposure to JP-8 fuel has been immunosuppression. $T$ cell responses such as delayed type hypersensitivity (DTH) reactions were greatly suppressed in mice by as little as a single application of $\mathrm{JP}-8^{[3]}$. Suppression of $\mathrm{T}$ cell proliferation was noted three days post exposure and continued for up to three weeks ${ }^{[3]}$. These effects seem to be associated with IL-10 production and appear to be abrogated utilizing anti-inflammatory treatments ${ }^{[9]}$.

While the immunosuppressive activity of JP-8 indicates that contact hypersensitivity might not result from exposure to this fuel, the DoD has identified that one of the main complaints of personnel exposed to the JP-8 fuel is "skin problems" (www.JP-8.org). While it suppresses DTH as stated above, JP-8 is well known to be proinflammatory, as it induces TNF $\alpha$ and IL- 8 in normal human keratinocyte cultures ${ }^{[10]}$. JP-8 causes skin barrier disruption, which leads to moderate to severe edema and erythema ${ }^{[11]}$, characterized by neutrophil influx in laboratory animal ${ }^{[12-14]}$. Extensive study of the very early events following JP-8 exposure found that numerous chemokines including CCL2, CCL3, CCL4, CXCL1 and CXCL2, as well as the cytokines IL-1 $\beta$ and IL-6, were variably induced up to eight hours post exposure ${ }^{[15]}$.

We previously described the modulation of multiple cytokines and chemokines following seven days of repeated dermal exposure to JP8 in rats $^{[14]}$. Herein, we expand on the previous study to examine cytokine and chemokine expression after one, three, five and seven days of repeated exposure. We find variable induction of various $\mathrm{CC}$ and $\mathrm{CXC}$ chemokines,

Corresponding Author:

Dr. Randle M. Gallucci, The University of Oklahoma Health Sciences Center, College of Pharmacy, Department of Pharmaceutical Sciences, P.O. Box 26901, Oklahoma City OK, 73190, Tel: 405-271-6593, Fax: 405-271-7477 
with a tendency toward production of neutrophil chemotractants, as well as the cytokines IL-1b and IL-6. Additionally, we find that protein expression of the inflammatory cytokine IL-6 is paradoxically modulated in skin by jet fuel exposure, which may contribute to cutaneous damage.

\section{MATERIALS AND METHODS}

Dermal JP-8 exposure: All animals received humane care according to the criteria outlined in the Guide for the Care and Use of Laboratory Animals prepared by the National Academy of Sciences and published by the National Institutes of Health (NIH publication 86-23, revised 1985). Male Long-Evans rats, 8-12 weeks old weighing approximately 200-250g, were acquired from Harlan (Harlan, Indianapolis, IN) and single housed in polycarbonate cages containing hardwood chip bedding at room temperature $\left(21 \pm 2^{\circ} \mathrm{C}\right)$ on a twelve-hour light/dark cycle. Animals were allowed to acclimate to the animal facility for at least one week prior to JP-8 exposure. One day prior to exposure, rats were sedated

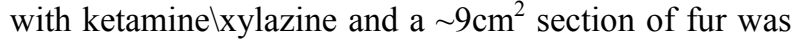
clipped and a depilatory (Nair) was applied to ensure complete removal of hair on the dorsal surface of the neck. Treatments were not initiated until at least 24 hours post hair removal, to ensure that no irritation occurred from the hair removal process. Immediately prior to exposure, treatment groups were placed in separate fume hoods to minimize exposure to JP-8 vapor. Filtered $(0.45 \mu \mathrm{m}) \mathrm{JP}-8$ (lot \# UN1863) was provided by the Air Force Research Laboratory (AFRL /HEPB) at Wright-Patterson Air Force Base and the Air Force Office for Scientific Research (AFOSR). In order to ensure consistent results, the fuel supplied by the AFOSR (lot \# UN1863) to all research labs requesting fuel for study is a homogenous mixture of multiple batches of fuel collected from various Air Force bases around the world. Jet fuel or acetone control $(300 \mu \mathrm{L})$ was applied to the neck of the animals and the groups were kept in fume hoods for one hour before being returned to the animal facility. Rats were treated as described for one, three, five, or seven days. Rat body weights were recorded daily and there were no significant differences with respect to treatment group. At 24 hours following a respective exposure, rats were anesthetized and skin samples were collected. Harvested skin was flash frozen in liquid nitrogen or fixed in $10 \%$ buffered formalin for histology.

Real-time quantitative PCR: Total RNA from rat skin was prepared and cDNA was synthesized using random decamer primers essentially as previously described ${ }^{[16]}$. Primers and probes for rat genes were purchased from Applied Biosystems (ABI, Foster City, CA). Real-time quantitative PCR was performed on an ABI PRISM 7000 SDS, utilizing TaqMan Master Mix according to the manufacturer's instructions (ABI). Quantitative values of genes of interest are normalized based on $28 \mathrm{~s}$ rRNA content.

Pro-inflammatory cytokine protein expression: Total protein from frozen skin samples was prepared by homogenizing skin samples in PBS $(10 \mathrm{mM}$ sodium phosphate, $150 \mathrm{mM} \mathrm{NaCl}, \mathrm{pH} 7.3$ ) containing protease inhibitor cocktail (\#P8340 Sigma, St. Louis, MO). Skin expression of proinflammatory cytokines was determined for TNF $\alpha$ and IL- 6 by commercially available ELISA kits (Biosource, Camarillo, CA) relative to total protein as determined by Bradford assay (BioRad, Hercules, CA).

Statistical Analysis: All experiments were replicated and representative findings are shown. Statistical significance was determined by one-way ANOVA. In all statistical comparisons, a $\mathrm{P}$ value of $<0.05$ was used to indicate a significant difference.

\section{RESULTS AND DISCUSSION}

Modulation of proinflammatory cytokine mRNA in JP-8 treated rat skin: The expression of various inflammatory cytokine genes was assessed by real-time quantitative PCR (QPCR) after one, three, five, or seven days of daily JP-8 application. Skin samples collected 24 hours following respective exposure were assessed for acute and chronic modulation of cytokine expression. The proinflammatory cytokines IL- $1 \beta$ and IL-6 were greatly upregulated beginning five days post exposure, approximately four and ten fold over control respectively (Fig. 1). IL-10 expression was not significantly modulated by JP-8 exposure at any time point (Fig. 1). Chemokines, which can be induced by primary proinflammatory cytokines like IL-1, were modulated quite variably. CCL3 was significantly induced at all time points (Fig. 2a), peaking at 15 fold over control on day five. CCL5 was only significantly increased after day one (Fig. 2a), where CCL11 was increased significantly after days one, five and seven (Fig. 2b). CCL20 was significantly induced only at the five day exposure (Fig. 2b). The chemokine CXCL1 was significantly induced after one (6 fold) and seven (3.5 fold) days, whereas CXCL2 was induced after three ( 4 fold) and five ( 8 fold) days (Fig. 3a). CXCL3 was progressively induced approximately 2-3 fold after three and five days, whereas CXCL10 was only significantly induced after 1 day (Fig. 3b).

Proinflammatory cytokine protein expression in JP8 treated rat skin: Keratinocyte cultures secrete various proinflammatory cytokines in response to JP-8 exposure $^{[10]}$ and we previously showed that chemokine protein expression followed mRNA expression ${ }^{[14]}$. However, there was shown a discrepancy concerning IL-6 expression in the skin after seven days of JP-8 exposure $^{[14]}$ and this protein is closely associated with 


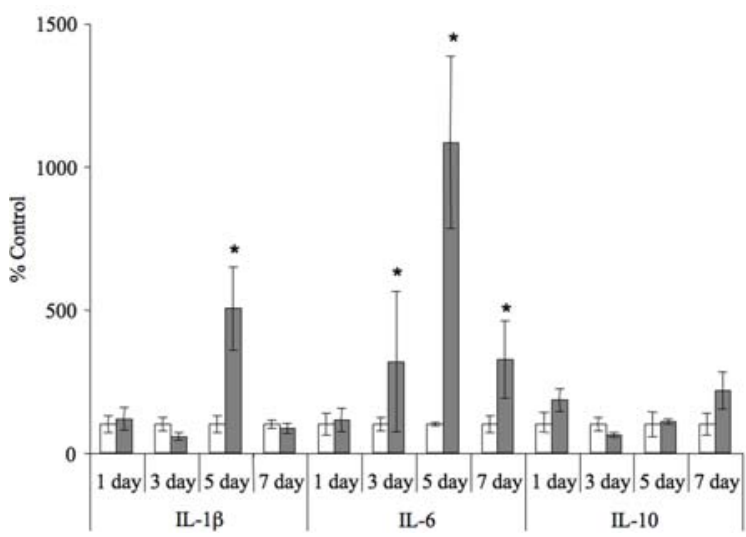

Fig. 1: IL-1 $\beta$, IL-6 and IL-10 mRNA modulation in skin following exposure to JP- 8 . Treatments $(300 \mu \mathrm{L}$ JP- $8=$ grey bars or acetone control $=$ white bars ) were applied to clipped skin of rats for up to seven days. Skin samples were collected after one, three, five, or seven days of treatment. Expression of IL-1 $\beta$, IL-6 and IL-10 mRNA's were analyzed by QPCR. Data are expressed as \% control acetone-treated treated skin and normalized to $28 \mathrm{~S}$ rRNA expression, $(+/-\mathrm{SE}, \mathrm{n}=10)$. *Significantly different from acetone control $(\mathrm{p} \leq 0.05)$

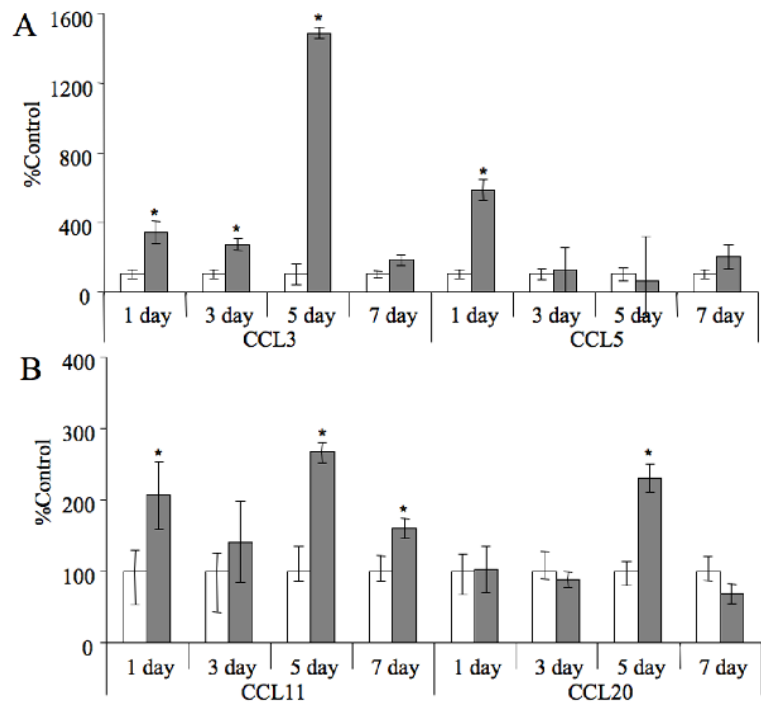

Fig. 2: Various C-C chemokine mRNA is significantly upregulated in skin following seven-day exposure to JP-8. Treatments $(300 \mu \mathrm{L}$ JP- $8=$ grey bars or acetone control $=$ white bars) were applied to clipped skin of rats for up to seven days. Skin samples were collected after one, three, five, or seven days of treatment. Expression of a) CCL3 and 5, or b) CCL11 and 20 mRNA's were analyzed by QPCR. Data are expressed as \% control acetone-treated treated skin and normalized to $28 \mathrm{~S}$ rRNA expression, $(+/-$ $\mathrm{SE}, \mathrm{n}=10)$. *Significantly different from acetone control $(\mathrm{p} \leq 0.05)$

skin healing ${ }^{[17]}$. To further investigate this, protein levels of IL- 6 and TNF $\alpha$ were determined by ELISA of skin homogenate after the indicated time periods of once daily JP-8 exposure. The skin levels of TNF $\alpha$ were found not to be different at any time point, confirming our and other's previous reports ${ }^{[14,15]}$

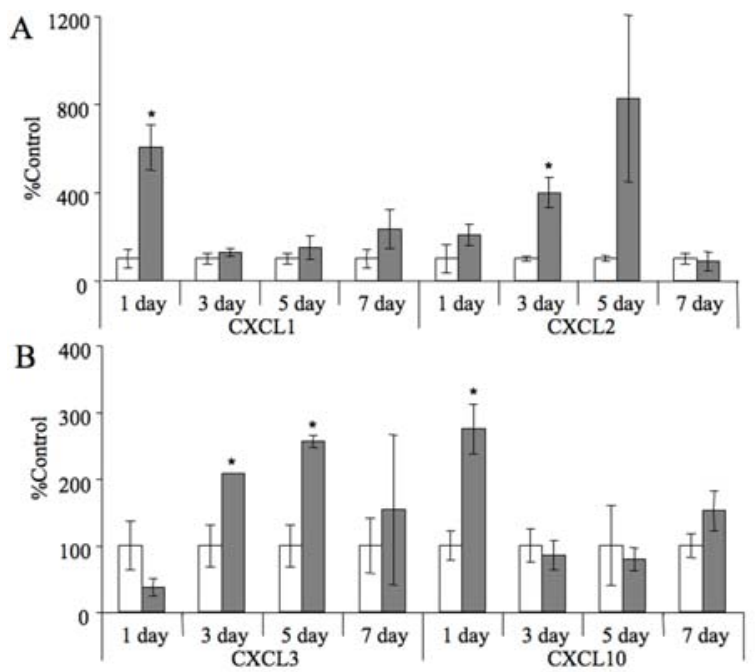

Fig. 3: Various C-X-C chemokine mRNA is significantly upregulated in skin following seven-day exposure to JP-8. Treatments $(300 \mu \mathrm{L} \mathrm{JP}-8=$ grey bars or acetone control $=$ white bars) were applied to clipped skin of rats for up to seven days. Skin samples were collected after one, three, five and seven days of treatment. Expression of a) CXCL1 and 2, or b) CXCL3 and 10 mRNA's were analyzed by QPCR. Data are expressed as \% control acetone-treated treated skin and normalized to 28S rRNA expression, (+/$\mathrm{SE}, \mathrm{n}=10)$. *Significantly different from acetone control $(\mathrm{p} \leq 0.05)$

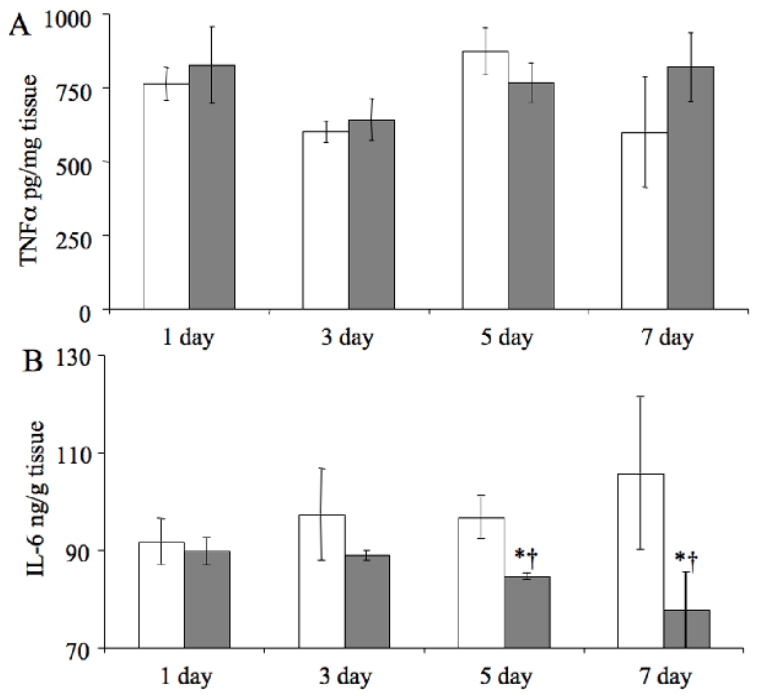

Fig. 4: Expression of IL-6 but not TNF $\alpha$ protein in skin is significantly induced by seven-day exposure to JP-8. Treatments $(300 \mu \mathrm{L}$ JP- $8=$ grey bars or acetone control $=$ white bars) were applied to clipped skin of rats for seven days. Skin samples were collected after one, three, five and seven days of treatment and total protein was isolated. Expression of (A) TNF $\alpha$ and (B) IL-6 protein was analyzed by commercial ELISA (BioSource, Camarillo, CA). Data are expressed as pg cytokine content per mg homogenized tissue extract, $(+/-\mathrm{SE}, \mathrm{n}=10)$. Significantly different from acetone control $(*)$, or one day JP-8 treated $(\dagger)(\mathrm{p} \leq 0.05)$

(Fig. 4a). However, IL-6 protein content in JP-8 treated skin appeared to decrease in a time wise fashion from one to seven days (Fig. 4b). 
In our previous article $e^{[14]}$, we presented data indicating that the cytokine expression profile seemed to support the observed acute inflammatory response that is dominant following JP-8 exposure. However, only one time point was investigated and only the end result was presented. Earlier events which might have shaped the response were not examined. In the present study, JP-8 was applied to an area of denuded rat skin for one, three, five and seven days and cytokine profiles were examined after each time point. It has recently been postulated that IL- $1 \alpha$ might be an early stimulus for the inflammatory cascade associated with JP-8 exposure. It is thought that damage to the epidermis associated with JP-8 exposure causes release of constitutively expressed IL- $1 \alpha$ and initiates a cascade of inflammatory cytokines that results in dermatitis ${ }^{[15]}$. Rapidly following an inflammatory stimulus, so-called "immediate early cytokines" such as TNF $\alpha$ and IL-1 $\beta$ were induced in numerous tissues ${ }^{[18]}$. We previously showed that both mRNA and protein for IL- $1 \beta$ but not TNF $\alpha$ was significantly induced following seven day exposure to JP-8. Herein, we show the kinetics of the IL-1 response to display a peak by five days that begins to decrease by seven (Fig. 1), which loosely correlates with overt dermatoses in JP-8 animals ${ }^{[12-14]}$. Interestingly, we did not report an increase in skin IL10 mRNA in our previous article and again we find this to be true throughout the treatment period (Fig. 1). This is of interest, as it is well known that IL-10 production as a result of JP-8 exposure leads to suppression of T cell responses ${ }^{[3]}$. However, it is entirely possible that this anti-inflammatory cytokine is produced at more distant sites, such as the draining lymph node and circulating levels of IL-10 were not measured during these experiments.

Chemokines, which are generally expressed following induction by one of the immediate early cytokines such as IL-1..are chemotactic cytokines and facilitate the trafficking of various inflammatory cells into a site of damage or infection. The CC chemokines, so called for the lack of intervening amino acids between a pair of conserved cysteines in its peptide sequence, tend to attract monocytes, eosinophils, basophils, immature dendritic cells and T lymphocytes to varying degrees ${ }^{[19]}$. While these cells tend to be involved in chronic or hypersensitivity reactions, other than monocytes, they are not observed in JP-8 induced lesions ${ }^{[14]}$. Indeed, it is well known that JP-8 decreases $\mathrm{DTH}$ and $\mathrm{T}$ cell responses ${ }^{[9]}$. Our previous work showed that seven-day JP-8 exposure resulted in the significant induction of CCL2, 3, 4 and $11^{[14]}$. To follow up on our previous study, we examine the expression of CCL3 3 and 11 as before, as well as CCL5 and 20. Herein, we find variable induction of these CC chemokines, where CCL3 is induced at all days but peaks at very high levels after five days, CCL5 is primarily induced after one day, CCL11 is progressively upregulated peaking at 7 days and CCL20 is not significantly induced (Fig. 2). The activities of CCL3, 5 and 11 are quite similar, all attracting eosinophil, macrophages, $\mathrm{T}$ cells, NK and immature dendritic cells with varying success (for review ${ }^{[20]}$ ). Based on the broad activity of these chemokines, it is difficult to associate their known specificities with the observed histological identification of JP-8 associated inflammatory cell infiltration. However, the specificity of CCL20 differs slightly, as it is known to attract primarily $\mathrm{B}$, mature $\mathrm{T}$ and immature dendritic cells ${ }^{[20]}$. The lack of significant expression of this particular chemokine does fit the observed cellular profile. Thus, while these chemokines can mediate the trafficking of cells other than PMN and macrophages, during JP-8 exposure, this activity does not manifest itself overtly. Why this might be is as yet uncertain.

As opposed to the $\mathrm{CC}$ chemokines, which favor cells other than PMN, CXC chemokines can be classified as either relatively strict neutrophil chemotractants or lymphocyte chemotractants, based on the type of receptor binding motif they possess. The presence of a glutamine-leucine-arginine motif (ELR+) in the cytokine peptide sequence seems to be associated with acute inflammation and PMN chemotaxis, whereas the ELR- type is associated with chronic inflammation (for review ${ }^{[20]}$ ). We previously reported that seven-day JP-8 exposure resulted in significant induction of the CXC chemokines CXCL1 and 2, which are neutrophil chemotractants, but not CXCL10, which is primarily a $\mathrm{T}$ cell chemotractant ${ }^{[14]}$. In the present study, we again find a tendency toward expression of PMN chemotractants, where CXCL1, 2 and 3 (ELR+) are increased in a time dependant fashion as a result of JP-8 exposure and CXCL10 (ELR-) is not (Fig. 3). It may be that increased ELR+ chemokine expression and the lack of increased expression of ELR- type chemokines can partially explain the transient nature of JP-8 induced irritant dermatitis. While not all CXC chemokines were examined, our data seems to indicate that PMN chemotactic chemokines such as CXCL1, 2 and 3 have a more profound effect on the pathogenesis of JP-8 skin dermatoses than those that are associated with chronic inflammation, such as many $\mathrm{CC}$ chemokines.

The cytokine IL-6 is also induced by immediate early inflammatory cytokines such as IL-1 and TNFa. In addition to its immunomodulatory activities, IL-6 is involved in the growth and differentiation of numerous cell types, including those of dermal and epidermal origin $^{[21]}$ and is closely linked to tissue regeneration and skin wound healing ${ }^{[17,22]}$. We previously reported that IL-6 mRNA was induced following seven-day JP-8 exposure, while data concerning protein expression was less clear ${ }^{[14]}$. In support of this previous data, we find that IL-6 mRNA is robustly induced in a timedependent manner (Fig. 1). 
However, when protein levels are examined there is an inverse correlation between the amount of IL-6 protein contained in the skin and length of JP-8 exposure (Fig. 4). The fate of IL-6 protein in JP-8 exposed skin is not clear. Certainly, JP-8 itself could be modulating the translation, secretion, or degradation of IL-6 in skin. Very little is known about the translation of IL-6 protein and even less is known concerning the effects JP-8 has on protein production. It could be that JP-8 is not affecting translation but in fact mediating rapid release of IL-6 from this skin. An increase in circulating IL-6 levels might account for this apparent change; however, this was not assessed in the present study. Interestingly, one of the primary routes of degradation of IL-6 is thought to occur in the skin ${ }^{[23,24]}$. It is tempting to speculate that perhaps JP-8 maybe be modulating metabolic or degradative pathways in the skin, since solvents can modulate proteasome activity ${ }^{[25,26]}$. However, at this point it is difficult to determine whether altered levels of IL-6 in skin are due to modulation of cytokine production or conversely, cytokine degradation, or if this phenomenon is unique to JP-8 exposure.

While the upregulation of proinflammatory cytokines and chemokines during JP-8 induced irritant dermatitis is confirmatory in nature, the apparent modulation of IL-6 by jet fuel appears to be a novel observation. At present, there are no reports investigating the expression of this cytokine following application of petroleum distillates to the skin. Since IL-6 is closely associated with skin wound healing, this observation could prove to be important where physical injury might occur in association with jet fuel exposure.

\section{ACKNOWLEDGEMENTS}

The authors wish to thank Ms. Lerin Luckett for her critical review of this manuscript. This work was funded by a grant from the Air Force Office of Strategic Research, \#02-NL023.

\section{REFERENCES}

1. Pfaff, J.K., B.J. Tollinger, R.C. Lantz, H. Chen, A.M. Hays and M.L. Witten, 1996. Neutral endopeptidase (NEP) and its role in pathological pulmonary change with inhalation exposure to JP-8 jet fuel. Toxicol. Ind. Health, 12: 93-103.

2. Harris, D.T., D. Sakiestewa, R.F. Robledo and M. Witten, 1997. Immunotoxicological effects of JP-8 jet fuel exposure. Toxicol. Ind. Health, 13: 43-55.

3. Ullrich, S.E., 1999. Dermal application of JP-8 jet fuel induces immune suppression. Toxicol. Sci., 52: 61-7.

4. Harris, D.T., D. Sakiestewa, D. Titone, R.S. Young and M. Witten, 2002. JP-8 jet fuel exposure results in immediate immunotoxicity, which is cumulative over time. Toxicol. Ind. Health, 18: 77-83.
5. Riviere, J.E., J.D. Brooks, N.A. Monteiro-Riviere, K. Budsaba and C.E. Smith, 1999. Dermal absorption and distribution of topically dosed jet fuels jet-A, JP-8 and JP-8(100). Toxicol. Appl. Pharmacol., 160: 60-75.

6. Pleil, J.D., L.B. Smith and S.D. Zelnick, 2000. Personal exposure to JP-8 jet fuel vapors and exhaust at air force bases. Environ. Health. Perspect., 108: 183-92.

7. McDougal, J.N., D.L. Pollard, W. Weisman, C.M. Garrett and T.E. Miller, 2000. Assessment of skin absorption and penetration of JP-8 jet fuel and its components. Toxicol. Sci., 55: 247-55.

8. Baynes, R.E., J.D. Brooks, K. Budsaba, C.E. Smith and J.E. Riviere, 2001. Mixture effects of JP-8 additives on the dermal disposition of jet fuel components. Toxicol. Appl. Pharmacol., 175: 26981.

9. Ullrich, S.E. and H.J. Lyons, 2000. Mechanisms involved in the immunotoxicity induced by dermal application of JP-8 jet fuel. Toxicol. Sci., 58: 2908.

10. Allen, D.G., J.E. Riviere and N.A. MonteiroRiviere, 2000. Identification of early biomarkers of inflammation produced by keratinocytes exposed to jet fuels jet A, JP-8 and JP-8(100). J. Biochem. Mol. Toxicol., 14: 231-7.

11. Kanikkannan, N., B.R. Locke and M. Singh, 2002. Effect of jet fuels on the skin morphology and irritation in hairless rats. Toxicology, 175: 35-47.

12. Monteiro-Riviere, N., A. Inman and J. Riviere, 2001. Effects of short-term high-dose and low-dose dermal exposure to Jet A, JP-8 and JP-8 +100 jet fuels. J. Appl. Toxicol., 21: 485-94.

13. Kabbur, M.B., J.V. Rogers, P.G. Gunasekar, C.M. Garrett, K.T. Geiss, W.W. Brinkley and J.N. McDougal, 2001. Effect of JP-8 jet fuel on molecular and histological parameters related to acute skin irritation. Toxicol. Appl. Pharmacol., 175: 83-8.

14. Gallucci, R.M., S.K. O'Dell, D. Rabe and L.D. Fechter, 2004. JP-8 jet fuel exposure induces inflammatory cytokines in rat skin. Int. Immunopharmacol., 4: 1159-69.

15. McDougal, J.N., C.M. Garrett, C.M. Amato and S.J. Berberich, 2006. Effects of brief cutaneous JP8 Jet fuel exposures on time-course of gene expression in the epidermis. Toxicol. Sci.

16. Simeonova, P.P. and M.I. Luster, 1995. Iron and reactive oxygen species in the asbestos-induced tumor necrosis factor-alpha response from alveolar macrophages. Am. J. Respir. Cell Mol. Biol., 12: 676-83.

17. Gallucci, R.M., P.P. Simeonova, J.M. Matheson, C. Kommineni, J.L. Guriel, T. Sugawara and M.I. Luster, 2000. Impaired cutaneous wound healing in interleukin-6-deficient and immunosuppressed mice. Faseb. J., 14: 2525-31. 
18. Luster, M.I., P.P. Simeonova, R.M. Gallucci, A. Bruccoleri, M.E. Blazka, B. Yucesoy, P. Simeonova, R. Gallucci, J. Matheson and T. Sugawara, 2001. Role of inflammation in chemical-induced hepatotoxicity Overview of immunotoxicology and current applications to respiratory diseases. Toxicol. Lett., 120: 317-21.

19. Alam, R., 1997. Chemokines in allergic inflammation. J. Aller. Clin. Immunol., 99: 273-7.

20. Baggiolini, M., B. Dewald and B. Moser, 1998. Chemokines and leukocyte traffic Human chemokines: An update. Nature, 392: 565-8.

21. Sehgal, P.B., 1990. Interleukin-6: molecular pathophysiology. J. Invest. Dermatol., $94 \quad(6$ Suppl.): 2S-6S.

22. Lin, Z.Q., T. Kondo, Y. Ishida, T. Takayasu and N. Mukaida, 2003. Essential involvement of IL-6 in the skin wound-healing process as evidenced by delayed wound healing in IL-6-deficient mice. J. Leukoc. Biol., 73: 713-21.

23. Castell, J., J. Klapproth, V. Gross, E. Walter, T. Andus, L. Snyers, J. Content and P.C. Heinrich, 1990. Fate of interleukin-6 in the rat. Involvement of skin in its catabolism. Eur. J. Biochem., 189: 113-8.
24. Rose-John, S., E. Hipp, D. Lenz, L.G. Legres, H. Korr, T. Hirano, T. Kishimoto and P.C. Heinrich, 1991. Structural and functional studies on the human interleukin-6 receptor. Binding, crosslinking, internalization and degradation of interleukin- 6 by fibroblasts transfected with human interleukin-6-receptor cDNA. J. Biol. Chem., 266: 3841-6.

25. Morishima, Y., H.M. Peng, H.L. Lin, P.F. Hollenberg, R.K. Sunahara, Y. Osawa and W.B. Pratt, 2005. Regulation of cytochrome P450 2E1 by heat shock protein 90-dependent stabilization and CHIP-dependent proteasomal degradation. Biochemistry, 44: 16333-40.

26. Osna, N.A., D.L. Clemens and T.M. Donohue, Jr., 2005. Ethanol metabolism alters interferon gamma signaling in recombinant HepG2 cells. Hepatology, 42: 1109-17. 